\section{GRAPHITE: UNSUPERVISED GRAPH EMBEDDINGS APPROACH TO MULTIPLEX IMMUNOFLUORESCENCE IMAGE EXPLORATION REVEALS NEW INSIGHTS INTO NSCLC AND HNSCC TUMOR MICROENVIRONMENT}

${ }^{1}$ Michael Surace ${ }^{*},{ }^{2}$ Helen Angell, ${ }^{2}$ Christopher Innocenti, ${ }^{2}$ Zhenning Zhang ${ }^{2}$ Isabelle Gaffney, ${ }^{2}$ Andreas Spitzmüller, ${ }^{2}$ Khan Baykaner, ${ }^{2}$ Balaji Selvaraj. ${ }^{1}$ Medimmune Inc., Gaithersburg, MD, United States; ${ }^{2}$ AstraZeneca, Baldock, United Kingdom

Background Predictive biomarkers for response to IO therapies remain insufficient. Although multiplex immunofluorescence has the potential to provide superior biomarkers, the information garnered from these studies is frequently underleveraged. Due to the large number of markers that must be analyzed (6 - $40+$ ), and the complexity of the spatial information, the number of hypotheses is large and must be tested systematically and automatically. GraphITE (Graphs-based Investigation of Tissues with Embeddings) is a novel method of converting multiplex IF image analysis results into embeddings, numerical vectors which represent the phenotype of each cell as well as the immediate neighborhood. This allows for the clustering of embeddings based on similarity as well as the discovery of novel predictive biomarkers based on both the spatial and multimarker data in multiplex IF images. Here we demonstrate initial observations from deployment of GraphITE on 564 commercially-sourced NSCLC and HNSCC resections stained with a multiplex IF panel containing CD8, PDL1, PD1, CD68, Ki67, and CK.

Methods $4 \mu \mathrm{m}$ FFPE tumor sections were stained with CD8, PDL1, PD1, CD68, Ki67, and CK at Akoya Biosciences using OPAL TSA-linked fluorophores and imaged on a Vectra Polaris. Images were analyzed by Computational Biology (AstraZeneca). Graphs were built by mapping each cell in the mIF image as a node, using the $\mathrm{X}, \mathrm{Y}$ coordinates and connecting nodes with edges according to distance. 64-dimensional embeddings were generated using Deep Graph InfoMax (DGI). ${ }^{1}$ Embeddings are downprojected to 2 dimensions using UMAP. $^{2}$. Details are available in the preprint of the GraphITE methods manuscript. ${ }^{3}$

Results A single downprojection was developed using embeddings from 158 HNSCC and 406 NSCLC cases. 60-80 distinct clusters were observed, some of which contained embeddings from both indications and others which were exclusive to one indication. Exclusive clusters describe tissue neighborhoods observed only in one indication. Drivers of cluster exclusivity included increased cell density in HNSCC as compared to NSCLC both in PD-L1- tumor centers with few infiltrating lymphocytes as well as in PD-L1- macrophagedominated neighborhoods. HNSCC and NSCLC embeddings were more colocalized in PD-L1+ tumor centers and in tumor stroma with high CD8+ or CD68+ immune cell content and high PD-L1+ expression.

Conclusions This study demonstrates the utility and potential of the GraphITE platform to discriminate between and describe both unique and common neighborhood-level features of the tumor microenvironment. Deploying GraphITE across multiple indications effectively leverages spatial heterogeneity and multimarker information from multiplex IF panels.

\section{REFERENCES}

1. Veličković $P$, Fedus W, Hamilton WL, Liò P, Bengio Y, DevonHjelm R. Deep Graph Infomax. 2018. arxiv:1809.10341 [stat.ML].

2. McInnes L, Healy J, Melville J. UMAP: Uniform manifold approximationand projection for dimension reduction. 2020; arxiv:1802.03426 [stat.ML].

3. Innocenti C, Zhang Z, Selvaraj B, Gaffney I, Frangos M, Cohen-Setton J, Dillon LAL, Surace MJ, Pedrinaci C, Hipp J, Baykaner K. An unsupervised graph embeddings approach to multiplex immunofluorescence image explorationbioRxiv 2021.06.09.447654; doi: https://doi.org/10.1101/2021.06.09.447654

Ethics Approval The study was approved by AstraZeneca.

http://dx.doi.org/10.1136/jitc-2021-SITC2021.822 